\title{
Time-Changed Poisson Processes
}

\author{
A. Kumar ${ }^{a}$, Erkan Nane ${ }^{b}$, and P. Vellaisamy ${ }^{a}$ \\ ${ }^{a}$ Department of Mathematics, Indian Institute of Technology Bombay, \\ Mumbai-400076, India. \\ ${ }^{b}$ Department of Mathematics and Statistics, Auburn University, \\ Auburn, AL 36849 USA.
}

\begin{abstract}
We consider time-changed Poisson processes, and derive the governing difference-differential equations (DDE) these processes. In particular, we consider the time-changed Poisson processes where the the time-change is inverse Gaussian, or its hitting time process, and discuss the governing DDE's. The stable subordinator, inverse stable subordinator and their iterated versions are also considered as time-changes. DDE's corresponding to probability mass functions of these time-changed processes are obtained. Finally, we obtain a new governing partial differential equation for the tempered stable subordinator of index $0<\beta<1$, when $\beta$ is a rational number. We then use this result to obtain the governing DDE for the mass function of Poisson process time-changed by tempered stable subordinator. Our results extend and complement the results in Baeumer et al. [3] and Beghin et al. [4] in several directions.

Key words: Hitting times; inverse Gaussian process; stable processes; time-changed process, subordination; tempered stable processes; difference-differential equation.
\end{abstract}

\section{Introduction}

Recently there has been an increasing interest to consider time-changed stochastic processes that yield solutions of fractional Cauchy problems, or solutions of higher order partial differential equations (PDE). The factional Cauchy problems can be used to model various phenomena in a wide range of scientific areas including physics, telecommunications, turbulence, image processing, biology, bioengineering, hydrology and finance, see [3, 4, 11, 18, 19]. There is an interesting connection between continuous time random walks and fractional Cauchy problems, see [20, 18, 19]

It is well known that the Poisson process $N(t)$ with parameter $\lambda>0$ solves the following difference-differential equation (DDE)

$$
\frac{d}{d t} p_{k}(t)=-\lambda p_{k}(t)+\lambda p_{k-1}(t),
$$


where $p_{k}(t)=\mathbb{P}\{N(t)=k\}$ is the probability mass function (pmf). The fractional Poisson process $N^{\beta}(t)$, which is a generalization of the Poisson process, solves the following fractional DDE (see e.g. Laskin (2003))

$$
\frac{d^{\beta}}{d t^{\beta}} p_{k}^{\beta}(t)=-\lambda p_{k}^{\beta}(t)+\lambda p_{k-1}^{\beta}(t),
$$

where $p_{k}^{\beta}(t)=\mathbb{P}\left\{N^{\beta}(t)=k\right\}$ and

$$
\frac{\partial^{\beta} u(t, x)}{\partial t^{\beta}}=\frac{1}{\Gamma(1-\beta)} \int_{0}^{t} \frac{\partial u(r, x)}{\partial r} \frac{d r}{(t-r)^{\beta}}
$$

for $0<\beta<1$, denotes the Caputo fractional derivative (see e.g. Caputo (1967)). Let $\tilde{u}(s, x)=\int_{0}^{\infty} e^{-s t} u(t, x) d t=\mathcal{L}_{t}(u(t, x))$ be the Laplace transform (LT) of $u(t, x)$ with respect to the variable $t$. Then Laplace transform is given by

$$
\mathcal{L}_{t}\left(\frac{\partial^{\beta} u(t, x)}{\partial t^{\beta}}\right)=s^{\beta} \tilde{u}(s, x)-s^{\beta-1} u(0, x) .
$$

The inverse Gaussian (IG) process $G(t)$ (or IG subordinator) has been found useful in financial modeling and is defined by (see Applebaum, 2009, p. 54)

$$
G(t)=\inf \{s>0 ; B(s)+\gamma s>\delta t\},
$$

where $B(t)$ is the standard Brownian motion. Note that $G(t) \sim \operatorname{IG}(\delta t, \gamma)$, the inverse Gaussian distribution with density

$$
g(x, t)=(2 \pi)^{-1 / 2}(\delta t) x^{-3 / 2} e^{\delta \gamma t-\frac{1}{2}\left(\frac{\delta^{2} t^{2}}{x}+\gamma^{2} x\right)}, \quad x>0 .
$$

Also, note that

$$
H(t)=\inf \{s \geq 0: G(s)>t\}
$$

denotes its first hitting time process.

In this paper, we consider the Poisson process time-changed by $G(t)$ and by the process $H(t)$, and investigate their properties. We consider also the problem of time-change by stable and tempered stable process, and derive the underlying PDE's. We only treat the case where the process and the time changes are assumed to be independent. Our results extend and complement the results in Baeumer et al. [3] and Orsingher et al. [4] in several directions.

\section{IG and its hitting time process as time-changes}

Let $N(t)$ be the Poisson process and $G(t)$ be the IG subordinator. First we consider the time-changed process $N(G(t))$. The probability mass function $\hat{p}_{k}(t)=\mathbb{P}(N(G(t))=k)$ of the 
time-changed process $N(G(t))$ is obtained by the standard conditioning argument as

$$
\begin{aligned}
\hat{p}_{k}(t) & =\int_{0}^{\infty} \frac{e^{-\lambda x}(\lambda x)^{k}}{k !}(2 \pi)^{-1 / 2}(\delta t) x^{-3 / 2} e^{\delta \gamma t-\frac{1}{2}\left(\frac{\delta^{2} t^{2}}{x}+\gamma^{2} x\right)} d x \\
& =\sqrt{\frac{2}{\pi}}(\delta t) e^{\delta \gamma t} \frac{\lambda^{k}}{k !}\left(\frac{\delta t}{\sqrt{\gamma^{2}+2 \lambda}}\right)^{k-1 / 2} K_{k-1 / 2}\left(\delta t \sqrt{\gamma^{2}+2 \lambda}\right)
\end{aligned}
$$

where $K_{\nu}(z)$ is the modified Bessel function of third kind with index $\nu$, defined by (see e.g. Abramowitz and Stegun (1992))

$$
K_{\nu}(\omega)=\frac{1}{2} \int_{0}^{\infty} x^{\nu-1} e^{-\frac{1}{2} \omega\left(x+x^{-1}\right)} d x, \omega>0
$$

Since, as $t \rightarrow \infty, \frac{N(t)}{t} \rightarrow \frac{1}{\lambda}$, a.s. and $\frac{G(t)}{t} \rightarrow \frac{\delta}{\gamma}$, a.s. (see e.g. Bertoin (1996), p. 92), we have

$$
\lim _{t \rightarrow \infty} \frac{N(G(t))}{t} \rightarrow \frac{\delta}{\lambda \gamma}, \text { a.s. }
$$

or equivalently $N(G(t)) \sim \frac{\delta}{\lambda \gamma} t$, a.s. This shows that the subordinated process does not explode in any finite interval of time.

Using the result (see Jørgenson (1992))

$$
\mathbb{E}(G(t))^{q}=\sqrt{\frac{2}{\pi}} \delta\left(\frac{\delta}{\gamma}\right)^{q-1 / 2} t^{q+1 / 2} e^{\delta \gamma t} K_{q-1 / 2}(\delta \gamma t)
$$

we easily obtain

$$
\mathbb{E} N(G(t))=\frac{\lambda \delta t}{\gamma} \text { and } \operatorname{Var}(N(G(t)))=\frac{\lambda \delta t}{\gamma}+\lambda^{2} \sqrt{\frac{2}{\pi}}(\delta t)\left(\frac{\delta t}{\gamma}\right)^{3 / 2} e^{\delta \gamma t} K_{3 / 2}(\delta \gamma t)-\left(\frac{\lambda \delta t}{\gamma}\right)^{2} .
$$

Remark 2.1. For the particular case $\delta=1$ and $\gamma=0$,

$$
\begin{aligned}
\mathbb{P}(N(G(t))=k) & =\int_{0}^{\infty} \frac{e^{-\lambda x}(\lambda x)^{k}}{k !} \frac{1}{\sqrt{2 \pi x^{3}}} t e^{-t^{2} / 2 x} d x \\
& \left.=\int_{0}^{\infty} \frac{e^{-\lambda t^{2} y}\left(\lambda t^{2} y\right)^{k}}{k !} \frac{1}{\sqrt{2 \pi y^{3}}} e^{-1 / 2 y} d x \quad \text { (put } x=t^{2} y\right) \\
& =\int_{0}^{\infty} \frac{e^{-\lambda t^{2} y}\left(\lambda t^{2} y\right)^{k}}{k !} f_{Y}(y) d y
\end{aligned}
$$

where $f_{Y}(y)=\frac{1}{\sqrt{2 \pi y^{3}}} e^{-1 / 2 y}, y>0$. Hence $N(G(t)) \stackrel{d}{=} N\left(\lambda t^{2} Y\right)$, a mixed Poisson process evaluated at time $t^{2}$. 
Remark 2.2. Note that $N(G(t))$ is not a renewal process, since here $G(t)$ is not an inverse subordinator (see Kingman (1964); Grandell (1976)). Indeed, it is the hitting time of $B(s)+\gamma s$, which is not a subordinator.

The density function $g(x, t)$ of $G(t)$ solves (see Kumar et al. (2011))

$$
\frac{\partial^{2}}{\partial t^{2}} g(x, t)-2 \delta \gamma \frac{\partial}{\partial t} g(x, t)=2 \delta^{2} \frac{\partial}{\partial x} g(x, t) .
$$

We have the following result.

Proposition 2.1. The pmf $\hat{p}_{k}(t)$ of the subordinated process $N(G(t))$ solves the following $D D E$ :

$$
\frac{d^{2}}{d t^{2}} \hat{p}_{k}(t)-2 \delta \gamma \frac{d}{d t} \hat{p}_{k}(t)=2 \delta^{2} \lambda\left[\hat{p}_{k}(t)-\hat{p}_{k-1}(t)\right] .
$$

Proof. We have

$$
\hat{p}_{k}(t)=\int_{0}^{\infty} p_{k}(x) g(x, t) d x .
$$

This implies by dominated convergence theorem

$$
\frac{d}{d t} \hat{p}_{k}(t)=\int_{0}^{\infty} p_{k}(x) \frac{\partial}{\partial t} g(x, t) d x \text { and } \frac{d^{2}}{d t^{2}} \hat{p}_{k}(t)=\int_{0}^{\infty} p_{k}(x) \frac{\partial^{2}}{\partial t^{2}} g(x, t) d x .
$$

Using the fact $\lim _{x \rightarrow \infty} g(x, t)=0=\lim _{x \rightarrow 0} g(x, t)$, we obtain

$$
\begin{aligned}
\left(\frac{d^{2}}{d t^{2}}-2 \delta \gamma \frac{d}{d t}\right) \hat{p}_{k}(t) & =\int_{0}^{\infty} p_{k}(x)\left(\frac{\partial^{2}}{\partial t^{2}}-2 \delta \gamma \frac{\partial}{\partial t}\right) g(x, t) d x \\
& =2 \delta^{2} \int_{0}^{\infty} p_{k}(x) \frac{\partial}{\partial x} g(x, t) d x \\
& =-2 \delta^{2} \int_{0}^{\infty} \frac{d}{d x} p_{k}(x) g(x, t) d x \\
& =-2 \delta^{2} \int_{0}^{\infty}\left[-\lambda p_{k}(x)+\lambda p_{k-1}(x)\right] g(x, t) d x \\
& =2 \delta^{2} \lambda\left[\hat{p}_{k}(t)-\hat{p}_{k-1}(t)\right],
\end{aligned}
$$

proving the result.

Next we consider the subordination of hitting time of the process $G(t)$. The right continuous first hitting time of the process $G(t)$ is defined by

$$
H(t)=\inf \{s \geq 0: G(s)>t\} .
$$

The process $H(t)$ has monotonically increasing continuous sample paths and it is not a Lévy process. The process $N(H(t))$ is a renewal process and the inter arrival times follows a 
tempered Mittag-Leffler distribution. Using Theorem 4.1 of Meerschaert et al. (2010), the process $N(H(t))$ is a renewal process whose iid waiting times $J_{n}$ satisfy

$$
\mathbb{P}\left(J_{n}>x\right)=E\left(e^{-\lambda H(x)}\right)
$$

and with LT

$$
\mathbb{E}\left(e^{-s J_{n}}\right)=\frac{\lambda}{\lambda+\delta\left(\sqrt{\gamma^{2}+2 s}-\gamma\right)} .
$$

For $\delta=1 / \sqrt{2}$, the rhs of the above equation reduce to

$$
\frac{\lambda}{\lambda+(s+a)^{\beta}-a^{\beta}},
$$

where $\beta=1 / 2$ and $a=\gamma^{2} / 2$. Also, the density of $J_{n}$ is

$$
f_{J_{n}}(x)=g(x) e^{-a x} \frac{\eta+a^{\beta}}{\eta},
$$

with

$$
g(x)=\frac{d}{d x}\left[1-E_{\beta}\left(-\eta x^{\beta}\right)\right] \text { and } \eta=\lambda-a^{\beta}
$$

(see Example 5.7 of Meerschaert et al. (2011)).

Remark 2.3. The condition (5.3) given in Meerschaert et. al.(2010) is satisfied by the process $G(t)$, since

$$
\begin{aligned}
\int_{0}^{1} x|\ln x| \pi(x) d x & =\int_{0}^{1} x|\ln x| \frac{\delta}{\sqrt{2 \pi x^{3}}} e^{-\gamma^{2} x / 2} d x \\
& \leq \frac{\delta}{\sqrt{2 \pi}} \int_{0}^{\infty} y^{-1 / 2}|\ln y| d y \\
& =\frac{\delta}{\sqrt{2 \pi}} \int_{0}^{\infty} z e^{-z / 2} d z=\frac{4 \delta}{\sqrt{2 \pi}}<\infty
\end{aligned}
$$

Thus their Theorem 5.2 is applicable on IG density.

Proposition 2.2. The pmf $\tilde{p}_{k}(t)=\mathbb{P}(N(H(t))=k)$ satisfies

$$
\begin{gathered}
\frac{d}{d t} \tilde{p}_{k}(t)=\frac{1}{2 \delta^{2}}\left[\left(\lambda^{2}-2 \delta \gamma \lambda\right) \tilde{p}_{k}(t)+\left(-2 \lambda^{2}+2 \delta \gamma \lambda\right) \tilde{p}_{k-1}(t)+\lambda^{2} \tilde{p}_{k-2}(t)\right]-\delta_{0}(t) \tilde{p}_{k}(0) \\
+\frac{1}{2 \delta^{2}} h(0, t) p_{k}^{\prime}(0)
\end{gathered}
$$

where $p_{k}^{\prime}(0)=-\lambda, \lambda$ and 0 for $k=0,1$ and $k \geq 2$ respectively. 
Proof. The density function $h(x, t)$ of $H(t)$ satisfies the following PDE (Vellaisamy and Kumar (2011))

$$
\frac{\partial^{2}}{\partial x^{2}} h(x, t)-2 \delta \gamma \frac{\partial}{\partial x} h(x, t)=2 \delta^{2} \frac{\partial}{\partial t} h(x, t)+2 \delta^{2} h(x, 0) \delta_{0}(t) .
$$

We have for $k \geq 0$

$$
\begin{aligned}
\frac{d}{d t} \tilde{p}_{k}(t) & =\int_{0}^{\infty} p_{k}(x) \frac{\partial}{\partial t} h(x, t) d x \\
& =\frac{1}{2 \delta^{2}} \int_{0}^{\infty} p_{k}(x)\left[\frac{\partial^{2}}{\partial x^{2}} h(x, t)-2 \delta \gamma \frac{\partial}{\partial x} h(x, t)\right] d x-\delta_{0}(t) \int_{0}^{\infty} p_{k}(x) h(x, 0) d x
\end{aligned}
$$

Now

$$
\begin{aligned}
\int_{0}^{\infty} p_{k}(x) \frac{\partial^{2}}{\partial x^{2}} h(x, t) d x & =\left.p_{k}(x) \frac{\partial}{\partial x} h(x, t)\right|_{0} ^{\infty}-\int_{0}^{\infty} p_{k}^{\prime}(x) \frac{\partial}{\partial x} h(x, t) d x \\
& =\left.p_{k}(x) \frac{\partial}{\partial x} h(x, t)\right|_{0} ^{\infty}-\left.h(x, t) p_{k}^{\prime}(x)\right|_{0} ^{\infty}+\int_{0}^{\infty} p_{k}^{(2)}(x) h(x, t) d x \\
& \left.=-2 \delta \gamma p_{k}(0) h(0, t)+h(0, t) p_{k}^{\prime} 0 x\right)+\int_{0}^{\infty} p_{k}^{(2)}(x) h(x, t) d x
\end{aligned}
$$

since $\lim _{x \rightarrow \infty} h_{x}(x, t)=0=\lim _{x \rightarrow \infty} h(x, t)$ (see Vellaisamy and Kumar (2011)). Also

$$
\begin{aligned}
\int_{0}^{\infty} p_{k}(x) \frac{\partial}{\partial x} h(x, t) d x & =\left.p_{k}(x) h(x, t)\right|_{0} ^{\infty}-\int_{0}^{\infty} p_{k}^{\prime}(x) h(x, t) d x \\
& =-p_{k}(0) h(0, t)-\int_{0}^{\infty} p_{k}^{\prime}(x) h(x, t) d x
\end{aligned}
$$

Using the fact $h_{x}(0, t)=2 \delta \gamma h(0, t)$, we get

$$
\begin{gathered}
\frac{d}{d t} \tilde{p}_{k}(t)=\frac{1}{2 \delta^{2}}\left[\int_{0}^{\infty} p_{k}^{(2)}(x) h(x, t) d x+2 \delta \gamma \int_{0}^{\infty} p_{k}^{\prime}(x) h(x, t) d x+h(0, t) p_{k}^{\prime}(0)\right] \\
-\tilde{p}_{k}(0) \delta_{0}(t) .
\end{gathered}
$$

The result now follows by substituting $p_{k}^{(2)}(x)=\lambda^{2}\left[p_{k}(x)-2 p_{k-1}(x)+p_{k-2}(x)\right]$ in (2.18) .

\section{Stable and inverse stable subordinators as time changes}

Let $f(x, t)$ be the density of a $\beta$-stable subordinator $D(t)$ with index $0<\beta<1$. Then the Laplace transform of $D(t)$ is given by

$$
\mathbb{E}\left(e^{-s D(t)}\right)=\int_{0}^{\infty} e^{-s x} f(x, t) d x=e^{-t s^{\beta}},
$$


where $s^{\beta}$ is called the Laplace exponent. The density $f(x, 1)$ of $D(1)$ is infinitely differentiable on $(0, \infty)$, with the asymptotics as follows: (see Uchaikin and Zolotarev (1999))

$$
\begin{gathered}
f(x, 1) \sim \frac{\left(\frac{\beta}{x}\right)^{\frac{2-\beta}{2(1-\beta)}}}{\sqrt{2 \pi \beta(1-\beta)}} e^{-(1-\beta)\left(\frac{x}{\beta}\right)^{-\frac{\beta}{1-\beta}}}, \quad \text { as } x \rightarrow 0 ; \\
f(x, 1) \sim \frac{\beta}{\Gamma(1-\beta) x^{1+\beta}}, \quad \text { as } x \rightarrow \infty .
\end{gathered}
$$

Note that from (3.1) and (3.2), we have

$$
\lim _{x \rightarrow 0} f(x, 1)=f(0,1)=0 \text { and } \lim _{x \rightarrow \infty} f(x, 1)=f(\infty, 1)=0 .
$$

Let $D^{*}(t)=\inf \{s \geq 0: B(s)>t / \sqrt{2}\}$ denote the first time when the Brownian motion (with variance $2 t$ ) hits the barrier $t$. The governing equation corresponding to the density function $g(x, t)$ of $D^{*}(t)$ can be obtained by putting $\delta=1 / \sqrt{2}$ and $\gamma=0$ in the equation (2.4). In this case, $D^{*}(t)$ is a stable subordinator of index $\frac{1}{2}$ with Laplace exponent $s^{1 / 2}$. We have the following proposition.

Proposition 3.1. Let $D_{1}^{*}, D_{2}^{*}, \cdots, D_{n}^{*}$ be independent $1 / 2$-stable subordinators. Then for the iterated composition defined by $D_{*}^{(n)}(t)=D_{1}^{*} o D_{2}^{*} o \cdots o D_{n}^{*}(t)$, the pmf $\tilde{p}_{k}(t)=P\left(N\left(D_{*}^{(n)}(t)\right)=\right.$ $k$ ), satisfies the following equation

$$
\frac{d^{2^{n}}}{d t^{2^{n}}} \tilde{p}_{k}(t)=\lambda\left[\tilde{p}_{k}(t)-\tilde{p}_{k-1}(t)\right]
$$

Proof. We prove this by induction. The case $n=1$ is obtained after putting $\delta=1 / \sqrt{2}$ and $\gamma=0$ in (2.5). Let $k(x, t)$ denote the density of $D_{1}^{*}(t)$, which can also be obtained by putting $\delta=1 / \sqrt{2}$ and $\gamma=0$ in (1.6). For the case $n=2$,

$$
\tilde{p}_{k}(t)=\int_{0}^{\infty} \int_{0}^{\infty} p_{k}\left(x_{1}\right) k\left(x_{1}, x_{2}\right) k\left(x_{2}, t\right) d x_{1} d x_{2} .
$$

Integrating by parts and then using (3.1) and (3.2), we get

$$
\begin{aligned}
\frac{d^{4}}{d t^{4}} \tilde{p}_{k}(t) & =\int_{0}^{\infty} \int_{0}^{\infty} p_{k}\left(x_{1}\right) k\left(x_{1}, x_{2}\right) \frac{\partial^{4}}{\partial t^{4}} k\left(x_{2}, t\right) d x_{1} d x_{2} \\
& =\int_{0}^{\infty} \int_{0}^{\infty} p_{k}\left(x_{1}\right) \frac{\partial^{2}}{\partial x_{2}^{2}} k\left(x_{1}, x_{2}\right) k\left(x_{2}, t\right) d x_{1} d x_{2} \\
& =\int_{0}^{\infty} \int_{0}^{\infty} p_{k}\left(x_{1}\right) \frac{\partial}{\partial x_{1}} k\left(x_{1}, x_{2}\right) k\left(x_{2}, t\right) d x_{1} d x_{2} \\
& =-\int_{0}^{\infty} \int_{0}^{\infty} \frac{d}{d x_{1}} p_{k}\left(x_{1}\right) k\left(x_{1}, x_{2}\right) k\left(x_{2}, t\right) d x_{1} d x_{2} \\
& =\lambda\left[\tilde{p}_{k}(t)-\tilde{p}_{k-1}(t)\right] .
\end{aligned}
$$

The general case also follows in similar way. 
Remark 3.1. (i) The composition of two stable subordinators is a stable subordinator. Let $D_{1}, D_{2}$ be two independent stable subordinators with Laplace exponents $c_{i} s^{\beta_{i}}, i=1,2$. Then

$$
\mathbb{E}\left[e^{-s D_{1}\left(D_{2}(t)\right)}\right]=E\left[e^{-c_{1} s^{\beta_{1}} D_{2}(t)}\right]=e^{-t c_{2} c_{1}^{\beta_{2}} s^{\beta_{1} \beta_{2}}},
$$

and hence $D_{1}\left(D_{2}(t)\right)$ is a stable subordinator of index $\beta_{1} \beta_{2}$. This implies that the composition $E_{1}\left(E_{2}(t)\right)$ is the inverse stable subordinator of index $\beta_{1} \beta_{2}$. Hence, by induction, we get

$$
E_{1}^{1 / 2}\left(E_{2}^{1 / 2}\left(E_{3}^{1 / 2}\left(\cdots E_{k}^{1 / 2}(t)\right)\right)\right)=E^{1 / 2^{k}}(t) .
$$

(ii) By Remark 2.1, we see that $D_{*}^{(n)}(t)$ is a stable subordinator of index $1 / 2^{n}$ and

$$
\mathbb{E}\left(e^{-s D^{(n)}(t)}\right)=\exp \left(-t s^{1 / 2^{n}}\right) .
$$

Note also also that $N\left(D^{(n)}(t)\right)$ is also a Lévy Process, as $N(t)$ is Poisson process and $D^{(n)}(t)$ is a stable subordinator (by Theorem 30.1 in Sato (1999)) and it has Laplace transform

$$
\mathbb{E}\left(e^{-s N\left(D^{(n)}(t)\right)}\right)=e^{-t(\log (\tilde{\mu}(s)))^{1 / 2^{n}}}=\exp \left(-t\left(\lambda\left(e^{-s}-1\right)\right)^{1 / 2^{n}}\right), s \geq 0
$$

where $\tilde{\mu}(s)=\mathbb{E}\left(e^{-s N(1)}\right)=\exp \left(\lambda\left(e^{-s}-1\right)\right)$. By Theorem 30.1 in Sato (1999), the Fourier exponent of $N\left(D^{(n)}(t)\right)$ can be written as

$$
\mathbb{E}\left(e^{i z N\left(D^{(n)}(t)\right)}\right)=\exp \left(-t\left(\lambda\left(e^{i z}-1\right)\right)^{1 / 2^{n}}\right), z \in \mathbb{R} .
$$

Remark 3.2. Let $0<\beta=\frac{k}{m}<1$ for $k, m$ relatively prime integers, and let $D(t)$ be a stable subordinator of index $\beta$ with $\mathbb{E}\left(e^{-s D(t)}\right)=e^{-c t s^{k / m}}$. In this case, the density $f(x, t)$ of $D(t)$ is a solution of

$$
\frac{\partial^{m}}{\partial t^{m}} f(x, t)=(-c)^{m} \frac{\partial^{k}}{\partial x^{k}} f(x, t), \quad x, t>0,
$$

see Lemma 3.1 in DeBlassie (2004). Hence, the density $k^{*}(x, t)$ of $D_{*}^{(n)}(t)$ satisfies

$$
\frac{\partial^{2^{n}}}{\partial t^{2^{n}}} k^{*}(x, t)=\frac{\partial}{\partial x} k^{*}(x, t), \quad x, t>0
$$

Alternative proof of Proposition 3.1 Using (3.8) one can obtain an equation for $\tilde{p}_{k}(t)=$ $\mathbb{P}\left(N\left(D_{*}^{(n)}(t)\right)=k\right)$ as follows:

$$
\begin{aligned}
\frac{d^{2^{n}}}{d t^{2^{n}}} \tilde{p}_{k}(t) & =\int_{0}^{\infty} p_{k}(x) \frac{\partial^{2^{n}}}{\partial t^{2^{n}}} k^{*}(x, t) d x \\
& =\int_{0}^{\infty} p_{k}(x) \frac{\partial}{\partial x} k^{*}(x, t) d x \\
& =\left.p_{k}(x) k^{*}(x, t)\right|_{0} ^{\infty}-\int_{0}^{\infty} k^{*}(x, t) \frac{\partial}{\partial x} p_{k}(x) d x \\
& =\lambda\left[\tilde{p}_{k}(t)-\tilde{p}_{k-1}(t)\right],
\end{aligned}
$$

which follows by integration by parts and using (3.1) and (3.2). 
Remark 3.3. Consider the standard Cauchy process $C(t)$ with density function

$$
q(x, t)=\frac{t}{\pi\left(x^{2}+t^{2}\right)}, x \in \mathbb{R} .
$$

The Cauchy process is a symmetric $\beta$-stable process with index $\beta=1$. Its Fourier transform (FT)

$$
\mathbb{E}\left(e^{-i u C(t)}\right)=\hat{q}(u, t)=e^{-t|u|} \text { or } \frac{\partial}{\partial t} \hat{q}(u, t)=-|u| \hat{q}(u, t)
$$

This implies

$$
\frac{\partial^{2}}{\partial t^{2}} \hat{q}(u, t)=|u|^{2} \hat{q}(u, t)=-(i u)^{2} \hat{q}(u, t)
$$

Invert the FT to get

$$
\frac{\partial^{2}}{\partial t^{2}} q(x, t)=-\frac{\partial^{2}}{\partial x^{2}} q(x, t)
$$

since $(i u)^{2} \hat{q}$ is the FT of $\partial^{2} q / \partial x^{2}$. The pmf $q_{k}(t)=P(N(|C(t)|)=k), k \geq 0, t>0$, satisfies the equation

$$
\begin{aligned}
\frac{d^{2}}{d t^{2}} q_{k}(t) & =-\lambda^{2}(1-\nabla)^{2} q_{k}(t)-\left.\frac{2}{\pi t} \frac{d}{d x} p_{k}(x)\right|_{x=0} \\
& =-\lambda^{2}(1-\nabla)^{2} q_{k}(t)+\left.\frac{2}{\pi t} \lambda(1-\nabla) p_{k}(x)\right|_{x=0},
\end{aligned}
$$

where $\nabla f_{k}(t)=f_{k-1}(t)$, the backward shift operator.

Let $f$ be in the domain of the generator, $-\lambda(1-\nabla)$, of the Poisson process, so that

$$
-\lambda(1-\nabla) f(k)=-\lambda(f(k)-f(k-1)) .
$$

Then $u(t, k)=\mathbb{E}_{k}(f(N(|C(t)|)))$ solves

$$
\begin{aligned}
\frac{d^{2}}{d t^{2}} u(k, t) & =-\lambda^{2}(1-\nabla)^{2} u(k, t)-\left.\frac{2}{\pi t} \frac{d}{d x} u(k, x)\right|_{x=0} \\
& =-\lambda^{2}(1-\nabla)^{2} u(k, t)+\frac{2}{\pi t} \lambda(1-\nabla) f(k), \\
u(0, k) & =f(k) .
\end{aligned}
$$

A general result that includes Equation (3.13) as a special case was first proved in Nane [21] We next look at the subordination to inverse stable subordinators. The inverse stable subordinator $E(t)$ ( of $D(t)$ of index $0<\beta<1$ ) has density

$$
\begin{aligned}
m(x, t) & =\frac{\partial}{\partial x} P(E(t) \leq x)=\frac{\partial}{\partial x}(1-P(D(x) \leq t)) \\
& =-\frac{\partial}{\partial x} \int_{0}^{\frac{t}{x^{1 / \beta}}} f(u, 1) d u=(t / \beta) f\left(t x^{-1 / \beta}, 1\right) x^{-1-1 / \beta}
\end{aligned}
$$


using the scaling property of the density $f(x, t)=(t / \beta) f\left(t x^{-1 / \beta}, 1\right) x^{-1-1 / \beta}$ (see Bertoin (1996)).

It is clear from (3.14) that the density $m(x, t)$ is in $C^{\infty}((0, \infty) \times(0, \infty))$. Some additional properties of $m(x, t)$ are given in the following Lemma whose proof follows from (3.1), (3.2), (3.14), and by taking Laplace transforms.

Lemma 3.1 (Lemma 2.1, Hahn et al (2010)). The density $m(x, t)$ of $E(t)$ satisfies

(a) $\lim _{t \rightarrow+0} m(x, t)=\delta_{0}(x)$ in the sense of the topology of the space of tempered distribution $\mathcal{D}^{\prime}(\mathbb{R})$;

(b) $\lim _{x \rightarrow+0} m(x, t)=\frac{t^{-\beta}}{\Gamma(1-\beta)}, t>0$;

(c) $\lim _{x \rightarrow \infty} m(x, t)=0, t>0$;

(d) $\int_{0}^{\infty} e^{-s t} m(x, t) d t=s^{\beta-1} e^{-x s^{\beta}}$.

Lemma 3.2 (Lemma 2.2, Hahn et al (2010); Theorem 4.1, Meerschaert and Scheffler (2008)). The density $m(x, t)$ is the fundamental solution of

$$
\frac{\partial^{\beta} m(x, t)}{\partial t^{\beta}}=-\frac{\partial m(x, t)}{\partial x}-\frac{t^{-\beta}}{\Gamma(1-\beta)} \delta_{0}(l)
$$

in the sense of tempered distributions.

Next, we consider the iterated composition of $H(t)$ for the case $\delta=1 / \sqrt{2}$ and $\gamma=0$. Define $H_{*}^{n}(t)=H_{1}^{*} o \cdots o H_{n}^{*}(t)$, where $H_{1}^{*}(t), \cdots, H_{n}^{*}(t)$ are $n$ independent copies of $H(t)$. Let $q_{k}(t)=$ $P\left(N\left(H_{*}^{n}(t)\right)=k\right)$. By Equation (3.6),$H_{*}^{n}(t)$ is the inverse of $D_{*}^{(n)}(t)$ with Laplace exponent $s^{1 / 2^{n}}$.

Remark 3.4. Let $E(t)$ be the inverse of a stable subordinator of index $1 / m$ then the density $m(x, t)$ of $E(t)$ satisfies

$$
\begin{aligned}
\frac{\partial m(x, t)}{\partial t} & =(-1)^{m} \frac{\partial^{m} m(x, t)}{\partial x^{m}}, \quad x, t>0 ; \\
\left.\frac{\partial^{k} m(x, t)}{\partial x^{k}}\right|_{x=0} & =t^{-(k+1) / m} \frac{(-1)^{k}}{\Gamma\left(1-\frac{(k+1)}{m}\right)}, \quad t>0, k=0,1,2, \cdots,(m-2) ; \\
\left.\frac{\partial^{m-1} m(x, t)}{\partial x^{m-1}}\right|_{x=0} & =0, \quad t>0 ; \\
\lim _{x \rightarrow \infty} \frac{\partial^{k} m(x, t)}{\partial x^{k}} & =0, \quad t>0, k=0,1,2, \cdots,(m-1) .
\end{aligned}
$$

The PDE (3.16) was obtained by Keyantuo and Lizama [14]. 
Using the last remark we can easily show

Theorem 3.1. Let $E(t)$ be inverse stable subordinator of index $0<\beta=1 / m<1$, for $m=2,3,4, \cdots$. Then the pmf $q_{k}(t)=\mathbb{P}(N(E(t))=k)$ solves the following $D D E$

$$
\frac{d}{d t} q_{k}(t)=(-\lambda)^{m}(1-\nabla)^{m} q_{k}(t)+\sum_{j=1}^{m-1}\left((-\lambda)^{j}(1-\nabla)^{j} p_{k}(0)\right) \frac{t^{-(m-j) / m}}{\Gamma\left(1-\frac{(m-j)}{m}\right)} .
$$

Proof of Theorem 3.1. This follows by using integration by parts and (3.16) above

$$
\begin{aligned}
\frac{d}{d t} q_{k}(t) & =\int_{0}^{\infty} p_{k}(x) \frac{\partial}{\partial t} m(x, t) d x \\
& =(-1)^{m} \int_{0}^{\infty} p_{k}(x) \frac{\partial^{m}}{\partial x^{m}} m(x, t) d x \\
& =\left.(-1)^{m} \sum_{j=1}^{m}(-1)^{j} \frac{\partial^{j-1}}{\partial x^{j-1}} p_{k}(x) \frac{\partial^{m-j}}{\partial x^{m-j}} m(x, t)\right|_{x=0}+\int_{0}^{\infty} \frac{\partial^{m}}{\partial x^{m}} p_{k}(x) m(x, t) d x \\
& =\left.\sum_{j=1}^{m}(-1)^{j} \frac{\partial^{j-1}}{\partial x^{j-1}} p_{k}(x)\right|_{x=0} t^{-(1+m-j) / m} \frac{(-1)^{j}}{\Gamma\left(1-\frac{(1+m-j)}{m}\right)}+\int_{0}^{\infty} \frac{\partial^{m}}{\partial x^{m}} p_{k}(x) m(x, t) d x \\
& =\left.\sum_{j=1}^{m} \frac{\partial^{j-1}}{\partial x^{j-1}} p_{k}(x)\right|_{x=0} \frac{t^{-(1+m-j) / m}}{\Gamma\left(1-\frac{(1+m-j)}{m}\right)}+\int_{0}^{\infty}(-\lambda)^{m}(1-\nabla)^{m} p_{k}(x) m(x, t) d x \\
& =\left.\sum_{j=2}^{m} \frac{\partial^{j-1}}{\partial x^{j-1}} p_{k}(x)\right|_{x=0} \frac{t^{-(1+m-j) / m}}{\Gamma\left(1-\frac{(1+m-j)}{m}\right)}+(-\lambda)^{m}(1-\nabla)^{m} q_{k}(t) \\
& =\left.\sum_{j=1}^{m-1} \frac{\partial^{j}}{\partial x^{j}} p_{k}(x)\right|_{x=0} \frac{t^{-(m-j) / m}}{\Gamma\left(1-\frac{(m-j)}{m}\right)}+(-\lambda)^{m}(1-\nabla)^{m} q_{k}(t)
\end{aligned}
$$

Note that the terms $\left.\frac{\partial^{j-1}}{\partial x^{j-1}} p_{k}(x)\right|_{x=0}$ might not be zero, in general. For example, $p_{k}(0)=1$ for $k=0, p_{k}(0)=0$ for $k \geq 1$. Also, $\left.\frac{\partial}{\partial x} p_{k}(x)\right|_{x=0}=0$ for $k \geq 2$, and it is equal to $\lambda$ for $k=1$ and is equal to $-\lambda$ for $k=0$.

Let $f$ be in the domain of the generator, $-\lambda(1-\nabla)$, of the Poisson process so that

$$
-\lambda(1-\nabla) f(k)=-\lambda(f(k)-f(k-1)) .
$$

Then $u(t, k)=\mathbb{E}_{k}(f(N(E(t))))$ solves

$$
\begin{aligned}
\frac{d}{d t} u(t, k) & =(-\lambda)^{m}(1-\nabla)^{m} u(t, k)+\sum_{j=1}^{m-1}\left((-\lambda)^{j}(1-\nabla)^{j} f(k)\right) \frac{t^{-(m-j) / m}}{\Gamma\left(1-\frac{(m-j)}{m}\right)} ; \\
u(0, k) & =f(k), k \geq 1
\end{aligned}
$$

This was first proved by Baeumer et al. (2009) [3] 
Corollary 3.1. The pmf $q_{k}(t)=\mathbb{P}\left(N\left(H_{*}^{n}(t)\right)=k\right)$ solves the following DDE

$$
\frac{d}{d t} q_{k}(t)=\lambda^{2^{n}}(1-\nabla)^{2^{n}} q_{k}(t)+\sum_{j=1}^{2^{n}-1}\left((-\lambda)^{j}(1-\nabla)^{j} p_{k}(0)\right) \frac{t^{-\left(2^{n}-j\right) / 2^{n}}}{\Gamma\left(1-\frac{\left(2^{n}-j\right)}{2^{n}}\right)},
$$

Remark 3.5. Let $E(t)$ be inverse stable subordinator of index $0<\beta<1$. Then the density $q_{k}(t)=\mathbb{P}(N(E(t))=k)$ solves (see Meerschaert et al. (2011)).

$$
\frac{d^{\beta}}{d t^{\beta}} q_{k}(t)=-\lambda(1-\nabla) q_{k}(t)
$$

In particular, when $\delta=1 / \sqrt{2}$ and $\gamma=0$, the density $q_{k}^{*}(t)=P\left(N\left(H_{*}^{n}(t)\right)=k\right)$ solves

$$
\frac{d^{1 / 2^{n}}}{d t^{1 / 2^{n}}} q_{k}^{*}(t)=-\lambda(1-\nabla) q_{k}^{*}(t), \quad n \geq 1
$$

Consider $\tilde{q}_{k}(t)=P\left(N\left(H^{n}(E(t))\right)=k\right)$. Since $H^{n}(E(t))$ is inverse stable subordinator of index $\beta / 2^{n}, \tilde{q}_{k}(t)$ solves

$$
\frac{d^{\beta / 2^{n}}}{d t^{\beta / 2^{n}}} \tilde{q}_{k}(t)=-\lambda(1-\nabla) \tilde{q}_{k}(t), \quad 0<\beta<1, n \geq 1
$$

Arguments similar to the ones above lead to the following result.

Proposition 3.2. The pmf $\tilde{q}_{k}(t)=\mathbb{P}\left(N\left(H^{n}(E(t))=k\right)\right.$ solves

$$
\frac{d^{\beta}}{d t^{\beta}} \tilde{q}_{k}(t)=\lambda^{2^{n}}(1-\nabla)^{2^{n}} \tilde{q}_{k}(t)+\sum_{j=1}^{2^{n}-1}\left(\left.\frac{\partial^{j}}{\partial z^{j}} p_{k}(z)\right|_{z=0}\right)\left[\frac{t^{-\beta\left(2^{n}-j\right) / 2^{n}} U\left(\left(2^{n}-j\right) / 2^{n}\right)}{\Gamma\left(1-\frac{\left(2^{n}-j\right)}{2^{n}}\right)}\right]
$$

where $U(\gamma)=\mathbb{E}\left[D(1)^{\gamma \beta}\right]$ for $0<\gamma<1$.

Proof. We can write

$$
\tilde{q}_{k}(t)=\mathbb{P}\left(N\left(H_{*}^{n}(E(t))\right)=k\right)=\int_{0}^{\infty} \mathbb{P}\left(N\left(H_{*}^{n}(x)\right)=k\right) m(x, t) d x=\int_{0}^{\infty} q_{k}^{*}(x) m(x, t) d x .
$$

Using the fact that the density $m(x, t)$ is the fundamental solution of

$$
\frac{\partial^{\beta} m(x, t)}{\partial t^{\beta}}=-\frac{\partial m(x, t)}{\partial x}-\frac{t^{-\beta}}{\Gamma(1-\beta)} \delta_{0}(x)
$$


in the sense of tempered distributions, we get

$$
\begin{aligned}
\frac{d^{\beta}}{d t^{\beta}} \tilde{q}_{k}(t) & =\int_{0}^{\infty} q_{k}^{*}(x) \frac{\partial^{\beta} m(x, t)}{\partial t^{\beta}} d x \\
& =\int_{0}^{\infty} q_{k}^{*}(x)\left[-\frac{\partial m(x, t)}{\partial x}-\frac{t^{-\beta}}{\Gamma(1-\beta)} \delta_{0}(x)\right] d x \\
& =-\int_{0}^{\infty} q_{k}^{*}(x) \frac{\partial m(x, t)}{\partial x} d x-q_{k}(0) \frac{t^{-\beta}}{\Gamma(1-\beta)} \\
& =-q_{k}^{*}(0) \frac{t^{-\beta}}{\Gamma(1-\beta)}-\left.q_{k}^{*}(x) m(x, t)\right|_{x=0} ^{\infty}+\int_{0}^{\infty} \frac{d q_{k}^{*}(x)}{d x} m(x, t) d x \\
& =-q_{k}^{*}(0) \frac{t^{-\beta}}{\Gamma(1-\beta)}+q_{k}^{*}(0) m(0, t) \\
& +\int_{0}^{\infty} m(x, t)\left[\lambda^{2^{n}}(1-\nabla)^{2^{n}} q_{k}(x)+\sum_{j=1}^{2^{n}-1}\left(\left.\frac{\partial^{j}}{\partial z^{j}} p_{k}(z)\right|_{z=0}\right) \frac{x^{-\left(2^{n}-j\right) / 2^{n}}}{\Gamma\left(1-\frac{\left(2^{n}-j\right)}{2^{n}}\right)}\right] d x \\
& =-q_{k}^{*}(0) \frac{t^{-\beta}}{\Gamma(1-\beta)}+q_{k}^{*}(0) \frac{t^{-\beta}}{\Gamma(1-\beta)}+\lambda^{2^{n}}(1-\nabla)^{2^{n}} \int_{0}^{\infty} m(x, t) q_{k}(x) d x \\
& +\sum_{j=1}^{2^{n}-1}\left(\left.\frac{\partial^{j}}{\partial z^{j}} p_{k}(z)\right|_{z=0}\right) \int_{0}^{\infty}\left[\frac{x^{-\left(2^{n}-j\right) / 2^{n}}}{\Gamma\left(1-\frac{\left(2^{n}-j\right)}{2^{n}}\right)}\right] m(x, t) d x \\
& =\lambda^{2^{n}}(1-\nabla)^{2^{n}} \tilde{q}_{k}(t)+\sum_{j=1}^{2^{n}-1}\left(\left.\frac{\partial^{j}}{\partial z^{j}} p_{k}(z)\right|_{z=0}\right) \mathbb{E}\left[\frac{(E(t))^{-\left(2^{n}-j\right) / 2^{n}}}{\Gamma\left(1-\frac{\left(2^{n}-j\right)}{2^{n}}\right)}\right] .
\end{aligned}
$$

We can calculate the terms $\mathbb{E}\left[E(t)^{-\gamma}\right]$ for $0<\gamma<1$ as follows: First $E(t) \stackrel{(d)}{=}(D(1) / t)^{-\beta}$ by Corollary 3.1 Meerschaert and Scheffler (2004), hence

$$
\mathbb{E}\left[E(t)^{-\gamma}\right]=\mathbb{E}\left[(D(1) / t)^{\gamma \beta}\right]=t^{-\beta \gamma} \mathbb{E}\left[D(1)^{\gamma \beta}\right]=: t^{-\beta \gamma} U(\gamma)<\infty
$$

Hence, we have

$$
\frac{d^{\beta}}{d t^{\beta}} \tilde{q}_{k}(t)=\lambda^{2^{n}}(1-\nabla)^{2^{n}-1} \tilde{q}_{k}(t)+\sum_{j=1}^{2^{n}-1}\left(\left.\frac{\partial^{j}}{\partial z^{j}} p_{k}(z)\right|_{z=0}\right)\left[\frac{t^{-\beta\left(2^{n}-j\right) / 2^{n}} U\left(\left(2^{n}-j\right) / 2^{n}\right)}{\Gamma\left(1-\frac{\left(2^{n}-j\right)}{2^{n}}\right)}\right]
$$

\section{Inverse of tempered stable processes as time-changes}

Note that tempered stable processes are obtained by exponential tempering in the distribution of stable processes, see Rosinski [22] for more details on tempering stable processes. The advantage of tempered stable process over stable process is that they are also infinitely divisible, 
and they have moments of all order. Let $f(x, t), 0<\beta<1$ denotes the density of a stable process (stable subordinator) with LT

$$
\int_{0}^{\infty} e^{-s x} f(x, t) d x=e^{-t s^{\beta}} .
$$

A tempered stable subordinator $D_{\mu}(t)$ has a density

$$
f_{\mu}(x, t)=e^{-\mu x+\mu^{\beta} t} f(x, t), \quad \mu>0 .
$$

The Lévy measure corresponding to a tempered stable process is given by (see e.g. Cont and Tankov (2004, p. 115))

$$
\pi_{D_{\mu}}(x)=\frac{c e^{-\mu x}}{x^{\beta+1}}, c>0, x>0
$$

which implies $\int_{0}^{\infty} \pi_{D_{\mu}}(x) d x=\infty$ and hence using Theorem 21.3 of Sato (1999), the sample paths of $D_{\mu}(t)$ are strictly increasing since jumping times are dense in $(0, \infty)$. Further the LT

$$
\tilde{f}_{\mu}(s, t)=\int_{0}^{\infty} e^{-s x} f(x, t) d x=e^{-t\left((s+\mu)^{\beta}-\mu^{\beta}\right)} .
$$

Proposition 4.1. The density function $f_{\mu}(x, t)$ of the tempered stable process of $D_{\mu}(t)$ satisfies the following PDE. For $\beta=\frac{1}{m}, m \geq 2$

$$
\sum_{j=1}^{m}(-1)^{j}\left(\begin{array}{c}
m \\
j
\end{array}\right) \mu^{(1-j / m)} \frac{\partial^{j}}{\partial t^{j}} f_{\mu}(x, t)=\frac{\partial}{\partial x} f_{\mu}(x, t) .
$$

Proof. We prove this result by induction. From (4.4) we have

$$
\tilde{f}_{\mu}(s, t)=e^{-t\left((s+\mu)^{\beta}-\mu^{\beta}\right)} .
$$

For $m=2$,

$$
\frac{\partial}{\partial t} \tilde{f}_{\mu}(s, t)=-\left((s+\mu)^{1 / 2}-\mu^{1 / 2}\right) \tilde{f}_{\mu}(s, t)
$$

and

$$
\frac{\partial^{2}}{\partial t^{2}} \tilde{f}_{\mu}(s, t)=\left((s+\mu)^{1 / 2}-\mu^{1 / 2}\right)^{2} \tilde{f}_{\mu}(s, t) .
$$

Using (4.7), (4.8) and using the fact that $f_{\mu}(0, t)=0$ (see (3.3) and (4.2) ), we get

$$
\left(\frac{\partial^{2}}{\partial t^{2}}-2 \mu^{1 / 2} \frac{\partial}{\partial t}\right) \tilde{f}_{\mu}(s, t)=s \tilde{f}_{\mu}(s, t)-f_{\mu}(0, t)
$$

Inverting the LT to get

$$
\left(\frac{\partial^{2}}{\partial t^{2}}-2 \mu^{1 / 2} \frac{\partial}{\partial t}\right) \tilde{f}_{\mu}(s, t)=\frac{\partial}{\partial x} \tilde{f}_{\mu}(s, t)
$$


Simililarily, for $m=3$

$$
\left(\frac{\partial^{3}}{\partial t^{3}}-3 \mu^{1 / 3} \frac{\partial^{2}}{\partial t^{2}}+3 \mu^{2 / 3} \frac{\partial}{\partial t}\right) \tilde{f}_{\mu}(s, t)=(-1)^{3} \frac{\partial}{\partial x} \tilde{f}_{\mu}(s, t) .
$$

The result also follows similarily for a general $m$.

Remark 4.1. Let $r_{k}(t)=P\left(N\left(D_{\mu}(t)\right)=k\right)$. Then the pmf $r_{k}(t)$ satisfies

$$
\sum_{j=1}^{m}(-1)^{j}\left(\begin{array}{c}
m \\
j
\end{array}\right) \mu^{(1-j / m)} \frac{d^{j}}{d t^{j}} r_{k}(t)=\lambda(1-\nabla) r_{k}(t) .
$$

Using the steps as the proof of Proposition 4.1., we can show that the density function $m_{\mu}(x, t)$ of the hitting time process $E_{\mu}(t)$ of $D_{\mu}(t)$ satisfies, for $\beta=\frac{1}{m}$ and $m \geq 2$,

$$
\sum_{j=1}^{m}(-1)^{j}\left(\begin{array}{c}
m \\
j
\end{array}\right) \mu^{(1-j / m)} \frac{\partial^{j}}{\partial x^{j}} m_{\mu}(x, t)=\frac{\partial}{\partial t} m_{\mu}(x, t)+\delta_{0}(t) m_{\mu}(x, 0) .
$$

When $\mu=0$, (4.12) reduces to

$$
(-1)^{m} \frac{\partial^{m}}{\partial x^{m}} m_{0}(x, t)=\frac{\partial}{\partial t} m_{0}(x, t)+\delta_{0}(t) m_{0}(x, 0)
$$

Let $E_{\mu}(t)$ denote the inverse of the tempered stable subordinator $D_{\mu}(t)$. By Theorem 3.1 in Meerschaert and Scheffler (2008) we have the density of $E_{\mu}(t)$ as

$$
m_{\mu}(x, t)=\int_{0}^{t} \pi_{D_{\mu}}(t-y, \infty) f_{\mu}(y, x) d y .
$$

Since the density $f_{\mu}(y, x)$ of $D_{\mu}(x)$ is infinitely differentiable, $m_{\mu}(x, t)$ is also infinitely differentiable.

Then we have the following result.

Proposition 4.2. The pmf $\tilde{r}_{k}(t)=P\left(N\left(E_{\mu}(t)\right)=k\right)$ satisfies the following PDE:

$$
\begin{array}{r}
\frac{d}{d t} \tilde{r}_{k}(t)=\sum_{j=1}^{m}\left(\begin{array}{c}
m \\
j
\end{array}\right) \mu^{(1-j / m)}(-\lambda(1-\nabla))^{j} \tilde{r}_{k}(t)+\sum_{j=1}^{m} \sum_{k=1}^{j}(-1)^{j+k}\left(\begin{array}{c}
m \\
j
\end{array}\right) \mu^{(1-j / m)} \\
\times\left.\frac{\partial^{k-1}}{\partial x k-1} p_{k}(x) \frac{\partial^{j-k}}{\partial x j-k} m_{\mu}(x, t)\right|_{x=0}-\delta_{0}(t) r_{k}(0) .
\end{array}
$$


Proof. We have

$$
\begin{aligned}
\frac{d}{d t} \tilde{r}_{k}(t)= & \int_{0}^{\infty} p_{k}(x) \frac{\partial}{\partial t} m_{\mu}(x, t) d x \\
= & \sum_{j=1}^{m}(-1)^{j}\left(\begin{array}{c}
m \\
j
\end{array}\right) \mu^{(1-j / m)} \int_{0}^{\infty} p_{k}(x) \frac{\partial^{j}}{\partial x^{j}} m_{\mu}(x, t) d x-\delta_{0}(t) r_{k}(0) \\
= & \sum_{j=1}^{m}(-1)^{j}\left(\begin{array}{c}
m \\
j
\end{array}\right) \mu^{(1-j / m)}\left[\left.\sum_{k=1}^{j}(-1)^{k} \frac{\partial^{k-1}}{\partial x^{k-1}} p_{k}(x) \frac{\partial^{j-k}}{\partial x^{j-k}} m_{\mu}(x, t)\right|_{x=0}\right. \\
& \left.\quad+(-1)^{j} \int_{0}^{\infty} \frac{d^{j}}{d x^{j}} p_{k}(x) m_{\mu}(x, t) d x\right]-\delta_{0}(t) r_{k}(0) \\
= & \left.\sum_{j=1}^{m} \sum_{k=1}^{j}(-1)^{j+k}\left(\begin{array}{c}
m \\
j
\end{array}\right) \mu^{(1-j / m)} \frac{\partial^{k-1}}{\partial x^{k-1}} p_{k}(x) \frac{\partial^{j-k}}{\partial x^{j-k}} m_{\mu}(x, t)\right|_{x=0} \\
& \quad+\sum_{j=1}^{m}(-1)^{j}\left(\begin{array}{c}
m \\
j
\end{array}\right) \mu^{(1-j / m)}(-\lambda(1-\nabla))^{j} r_{k}(t)-\delta_{0}(t) r_{k}(0) .
\end{aligned}
$$

Hence the result.

\section{References}

[1] Abramowitz, M. and Stegun, I. A. (eds) (1992). Handbook of Mathematical Functions with Formulas, Graphs and Mathematical Tables. Dover, New York.

[2] Applebaum, D. (2009). Lévy Processes and Stochastic Calculus. 2nd ed., Cambridge University Press, Cambridge, U.K.

[3] Baeumer, B. Meerschaert, M.M. and Nane, E. (2009) Brownian subordinators and fractional Cauchy problems. Trans. Amer. Math. Soc. 361, 3915-3930.

[4] Beghin, L. and Orsingher, E. (2009) Fractional Poisson processes and related planar random motions. Electron. J. Probab. 14 , no. 61, 17901827.

[5] Bertoin, J. (1996). Lévy Processes. Cambridge University Press, Cambridge.

[6] Bochner, S. (1955). Harmonic Analysis and the Theory of Probability. Univ. California press, Berkeley. CA.

[7] Caputo, M. (1967). Linear models of dissipation whose Q is almost frequency independent. Part II. Geophys. J. R. Astr. Soc. 13 529-539. 
[8] Cont, R. and Tankov, P. (2004). Financial Modeling with Jump Processes. Chapman \& Hall, CRC Press, Boca Raton.

[9] DeBlassie R. D. (2004), Higher order PDE's and symmetric stable processes, Probab. Theory Relat. Fields. 129, 495-536.

[10] Grandell, J. (1976). Doubly Stochastic Poisson Processes. Lecture Notes Math. 529. Springer, Berlin.

[11] Hahn, M. G., Kobayashi, K. and Umarov, S. (2011). Fokker-plank-Kolmogorov equations associated with time-changed fractional Brownian motion. Proce. Amer. Math. Soc. 139, 691-705.

[12] Halgreen, C. (1979). Self-decomposability of the generalized inverse Gaussian and hyperbolic distributions. Z. Wahrsch. Verw. Gebiete. 47, 13-17.

[13] Jeanblanc, M., Yor, M. and Chesney, M. (2009). Mathematical Methods for Financial Markets. Springer-Verlag, London.

[14] Keyantuo, V. and Lizama, C. (2009). On a connection between powers of operators and Fractional Cauchy problems. URL: http://netlizama.usach.cl/Keyantuo-Lizama(AMPA)(2009).pdf

[15] Kingman, J. F. C. (1964). On the doubly stochastic Poisson processes. Proc. Camb. Phil. Soc. 60, 923-930.

[16] Kumar, A., Meerschaert, M. M. and Vellaisamy, P. (2011). Fractional normal inverse Gaussian diffusion. Statist. Probab. Lett. 81, 146-152.

[17] Laskin, N. (2003). Fractional Poisson process. Commun. Nonlinear Sci. Numer. Simul., 8, 301-213.

[18] Meerschaert, M. M. and Scheffler, H. (2004). Limit theorems for continuous-time random walks with infinite mean waiting times. J. App. Prob. 41, 623-638.

[19] Meerschaert, M. M. and Scheffler, H. (2008). Triangular array limits for continuous time random walks. Stochastic Process. Appl. 118, 1606-1633.

[20] Meerschaert, M. M., Nane, E. and Vellaisamy, P. (2011). The fractional Poisson process and the inverse stable subordinator. Communicated.

[21] Nane, E. (2008). Higher order PDE's and iterated processes. Trans. Amer. Math. Soc. 360, 2681-2692. 
[22] J. Rosiński, Tempering stable processes. Stochastic Process Appl. 117 (2009), 677-707.

[23] Sato, K.I. (1999). Lévy Processes and Infinitely Divisible Distributions. Cambridge University Press.

[24] Steutel, F.W. and Van Harn, K. (2004). Infinite Divisibility of Probability Distributions on the Real Line. Marcel Dekker, New York.

[25] Vellaisamy, P. and Kumar, A. (2011). The hitting time of inverse Gaussian process. Preprint.

[26] Uchaikin, V. V. and Zolotarev, V. M. (1999). Chance and Stability: Stable Distributions and Their Applications. VSP. Utrecht.

\section{Notation}

$$
\begin{array}{rr}
\text { variable } & \text { density } \\
G(t) & g(x, t) \\
\delta=1 / \sqrt{2}, \gamma=0 & k(x, t) \\
H(t) & h(x, t) \\
\delta=1 / \sqrt{2}, \gamma=0 & l(x, t) \\
\beta-\text { stable } D(t) & f(x, t) \\
\beta / 2-\text { stable } Y(t) & p(x, t) \\
1 / 2-\text { stable } n^{*} & k^{*}(x, t) \\
E(t) & m(x, t) \\
\delta=1 / \sqrt{2}, \gamma=0 & l(x, t) \\
1 / 2-\text { inverse stable } n^{*} & l^{*}(x, t) \\
\text { Caucy } C(t) & q(x, t) \\
\text { TS } D_{\mu}(t) & f_{\mu}(x, t) \\
{\text { Hittingtime } E_{\mu}(t)}(x, t)
\end{array}
$$

